\title{
Knowledge of Cervical Cancer and Human Papillomavirus Vaccines among Child-Bearing Aged Women in Hanoi, Vietnam
}

\author{
Nguyen Thi Ngoc Phuong ${ }^{1,2}$, Le Thi Thanh Xuan², Le Thi Huong², Do Thi Thanh \\ Toan $^{2}$, Jin Kyung Oh${ }^{1}$, Young Joo Won ${ }^{3}$, Kui Son Choi ${ }^{1 *}$
}

\begin{abstract}
Objectives: The study sought to examine knowledge of cervical cancer and human papillomavirus (HPV) vaccines among child-bearing aged women in Hanoi, Vietnam. Methods: In 2016, 807 women aged 18 to 49 years were recruited from one urban area and one rural area in 2016 and were examined through face-to-face paper-based interviews. Pearson's chi-square test and an independent t-test were utilized to compare awareness of cervical cancer and HPV vaccination among women according residential status. Results: Overall, $83.8 \%$ and $71.3 \%$ women had heard about cervical cancer and HPV vaccination, respectively. Mean knowledge scores for cervical cancer and HPV vaccination were $4.60 \pm 1.43$ out of 7 and $1.53 \pm 1.35$ out of 5 , respectively. Women living in an urban area were more likely to be aware of cervical cancer and to be more knowledgeable of HPV vaccination than women in a rural area. Conclusions: Despite strong awareness, we found knowledge on cervical cancer and HPV vaccination to be alarmingly insufficient among Vietnamese women.
\end{abstract}

Keywords: Cervical cancer- HPV vaccines- knowledge- Vietnam

Asian Pac J Cancer Prev, 21 (7), 1951-1957

\section{Introduction}

Cervical cancer poses a tremendous global health challenge, especially in less developed parts of the world where cervical cancer incidence and mortality rates can reach up to $80 \%$ (Bray et al.; World Health Organization, 2016). Safe sex behavior, getting an HPV vaccine, and cervical cancer screening are well-recognized ways to successfully prevent this cancer and lower mortality rates (World Health Organization, 2016). Since its launch in 2006, HPV vaccination has garnered consideration as an important life-saving intervention for millions of women throughout the world. Indeed, in the most developed parts of the world, where cervical cancer screening and HPV vaccination are readily available, cervical cancer incidence rates have dropped dramatically (Vaccarella et al., 2013).

In Vietnam, cervical cancer ranks as the second most common cancer among women of ages ranging from 15 to 44 years (Bruni, 2017). To date, cervical cancer screening to detect cancer early is only offered in opportunistic screening programs in Vietnam, and few women elect to undergo cervical cancer screening using their own financial resources. Also, according to a report in 2016 by the United Nations, condom usage among men in Vietnam was extremely low, at less than $12 \%$ (United Nations,
2016). To prevent cervical cancer in Vietnam, a pilot program for HPV vaccination was implemented in 2008; however, until now, it has not been incorporated into the national immunization program. Moreover, with limited resources dedicated to the prevention of cervical cancer, raising awareness of cervical cancer and HPV vaccination has proven to be a major challenge (Bingham et al., 2009; Tung et al., 2016).

The aim of this study was to examine knowledge of cervical cancer and HPV vaccination among women of child-bearing age in Hanoi, Vietnam using data collected in 2016. Understanding these data will help with designing and implementing successful vaccination programs through which to propel acceptance of HPV vaccination forward in Vietnamese society and minimizing the burden of this disease.

\section{Materials and Methods}

\section{Data sources and Setting}

Data were obtained from a project funded by Pfizer, an American pharmaceutical company, entitled "Vaccination accessibility for child-bearing age women in low- and middle-income countries in Southeast Asia period 2016-2018," which was conducted 
as a multi-country, pre- and post-evaluation, and community-based randomized control trial for improving awareness and utilization of several vaccine types (e.g., rubella, influenza, tetanus, hepatitis B, and HPV) among reproductive-aged women. In the current study, data collected for pre-evaluation were utilized.

\section{Study design and sample}

The present study was conducted as an observational cross-sectional study with a convenient multistage cluster sampling method. First, two districts, representative of residence status, were randomly chosen: Dong Da (urban area) and $\mathrm{Ba} \mathrm{Vi} \mathrm{(rural} \mathrm{area).} \mathrm{Second,} \mathrm{two} \mathrm{health} \mathrm{communes}$ were randomly selected for these two areas. Finally, lists of all women who were pregnant or had just given birth in the last 12 months at the time of the survey were received from the local health centers.

The potential participants were recruited in this study if they met the following inclusion criteria: (1) women aged 18 to 49 years at the time of the survey; (2) currently living in the selected communes for at least 1 year and presence during the recruitment times; (3) pregnant or gave birth within the last 12 months (from April 16, 2015 to April $15,2016)$; and (4) agreed to participate in this project.

Regarding sample size calculation, since no study has measured coverage of HPV vaccination among reproductive-aged women in Vietnam, we set $\mathrm{P}$ to equal 0.5 . With $95 \%$ confidence intervals, a maximum permissible error $(\Delta)$ of 0.05 and a $10 \%$ drop out in case an individual refused to participate or was absent during the collection period, final sample sizes of 400 women in each district and 800 in total were calculated. Participants were then selected from two communal health units in Dong $\mathrm{Da}$ and $\mathrm{Ba}$ Vi from lists of 975 and 470 eligible women, respectively. A total of 807 women finally participated in this study.

\section{Study instrument}

Face-to-face interviews were conducted by a professional researcher using a structured questionnaire. Guidelines from the Vietnam Ministry of Health and relevant references from the National Institute of Hygiene and Epidemiology were utilized develop this questionnaire (Vietnam Ministry of Health, 2009). This questionnaire was reviewed by five experts and pre-tested by five pregnant women and five child-bearing women within a year before actual implementation.

The questionnaire comprised three parts: sociodemographic information and awareness and knowledge of cervical cancer and HPV vaccination. Awareness of cervical cancer and HPV vaccination was assessed by items concerning whether the interviewee had ever heard of cervical cancer or HPV vaccination, responding either yes or no. For those who had heard about either cervical cancer or HPV vaccination, detailed questions on cervical cancer (7 questions) or HPV vaccination (5 questions) were then asked with possible responses of true, false, unknown, or no response. Sometimes the correct answer to the question was "true," and other times it was "false." One point was given for each correct answer, and points were summed to create a summary knowledge score. If a question had more than one correct answer, 1 point was given for at least one correct answer. Higher knowledge scores indicated having greater knowledge of cervical cancer or HPV vaccination. All summary knowledge scores were divided into two groups (good and not good) according to mean scores described in a previous study (Prayudi et al., 2016). Beyond the mean scores, we further considered the distribution of knowledge scores (supplementary material) to identify an appropriate cut-off point (median value) for categorical decisions. Levels of knowledge of cervical cancer were categorized as "good" if more than five out of the seven questions were answered correctly or as "not good" if not. Levels of HPV knowledge were classified as "good" if more than two out of the five questions were answered correctly or as "not good" if not. Health status was self-reported by the participants using the scale of good, neutral, or not good.

All participants provided informed consent after explaining the survey's objectives, that participation was voluntary, the confidentiality of this survey, the procedure, and potential benefits for the individual and communities. Only those who agreed to participate in this survey and provided consent were included in the study. The surveys were administered in either a health commune or a participant's home upon request. Each participant took about 30-40 minutes to complete the interview and received a gift $(50,000 \mathrm{VND})$ for their time spent.

\section{Ethical Review}

Ethical approval from the Institutional Ethical Review Board of the Institute for Preventive Medicine and Public Health, Hanoi Medical University in Hanoi, Vietnam (No 184/HMU IRB) was obtained for the study. Verbal informed consent was obtained from all participants.

\section{Data analysis}

All completed questionnaires were entered into EpiData version 3.1 (EpiData Association, Denmark) independently by two people to ensure data accuracy. Descriptive analysis was conducted for socio-demographic factors and awareness and knowledge of cervical cancer and HPV vaccines. Pearson's chi-square test and Fisher's exact test were employed for bivariate analysis of all categorical variables, whereas an independent t-test was used to test differences in mean knowledge scores between women living in the two residential areas. All P values less than 0.05 were considered to be indicative of statistical significance. All statistical analyses were performed using STATA software, version 14 (Stata Corp. L.P., College Station, TX).

\section{Results}

The demographical characteristics of the respondents are presented in Table 1. Of the 807 participants, about two-thirds (624 women) were younger than 30 years, and more younger women tended to reside in the rural area $(\mathrm{Ba}$ Vi) than in the urban area (Dong Da). Overall, women with less education and blue-collar workers or peasants more frequently resided in the rural area. While health insurance coverage almost doubled among women living in the 
DOI:10.31557/APJCP.2020.21.7.1951

Knowledge of Cervical Cancer and Human Papillomavirus Vaccines among Child-Bearing Aged Women in Hanoi, Vietnam Table 1. Demographic Characteristic

\begin{tabular}{|c|c|c|c|c|c|c|c|}
\hline \multirow[t]{2}{*}{ Variables } & \multicolumn{2}{|c|}{ Overall } & \multicolumn{2}{|c|}{ Urban } & \multicolumn{2}{|c|}{ Rural } & \multirow[t]{2}{*}{$P$-value } \\
\hline & $\mathrm{n}$ & $\%$ & $\mathrm{n}$ & $\%$ & $\mathrm{n}$ & $\%$ & \\
\hline Total & 807 & 100 & 400 & 49.6 & 407 & 50.4 & \\
\hline \multicolumn{8}{|l|}{ Age group } \\
\hline$<=25$ years old & 222 & 27.5 & 64 & 16 & 158 & 38.8 & $<0.001$ \\
\hline 26-30 years old & 302 & 37.4 & 156 & 39 & 146 & 35.9 & \\
\hline$>30$ years old & 283 & 35.1 & 180 & 45 & 103 & 25.3 & \\
\hline \multicolumn{8}{|l|}{ Marital status } \\
\hline With spouse & 801 & 99.3 & 398 & 99.5 & 403 & 99.0 & 0.43 \\
\hline Without spouse & 6 & 0.7 & 2 & 0.5 & 4 & 1.0 & \\
\hline \multicolumn{8}{|l|}{ Child-birth status } \\
\hline Having infants ( $0-1$ years) & 609 & 75.5 & 299 & 74.8 & 310 & 76.2 & 0.64 \\
\hline Pregnant & 198 & 24.5 & 101 & 25.2 & 97 & 23.8 & \\
\hline \multicolumn{8}{|l|}{ Residential status } \\
\hline Permanent residence & 706 & 87.5 & 320 & 80 & 386 & 94.8 & $<0.001$ \\
\hline Temporary residence & 101 & 12.5 & 80 & 20 & 21 & 5.2 & \\
\hline \multicolumn{8}{|l|}{ Educational attainment } \\
\hline College or higher & 424 & 55.5 & 322 & 80.5 & 102 & 25.1 & $<0.001$ \\
\hline High school or lower & 383 & 47.5 & 78 & 19.5 & 305 & 74.9 & \\
\hline \multicolumn{8}{|l|}{ Occupation\# } \\
\hline White-collar & 252 & 31.2 & 213 & 53.3 & 39 & 9.6 & $<0.001$ \\
\hline Blue-collar/ Peasants & 284 & 35.2 & 16 & 4 & 268 & 65.9 & \\
\hline Others & 271 & 33.6 & 171 & 42.7 & 100 & 24.5 & \\
\hline \multicolumn{8}{|c|}{ Household monthly income per person* } \\
\hline Moderate or higher & 598 & 74.1 & 382 & 95.5 & 216 & 53.1 & $<0.001$ \\
\hline Poor or near poor & 209 & 25.9 & 18 & 4.5 & 191 & 46.9 & \\
\hline \multicolumn{8}{|l|}{ Health insurance card } \\
\hline Yes & 509 & 63.1 & 316 & 79 & 193 & 47.4 & $<0.001$ \\
\hline No/No response & 298 & 36.9 & 84 & 21 & 214 & 52.6 & \\
\hline \multicolumn{8}{|l|}{ Self-reported health status } \\
\hline Good & 502 & 62.2 & 255 & 63.8 & 247 & 60.7 & $<0.001$ \\
\hline Neutral or not good & 305 & 37.8 & 145 & 36.2 & 160 & 39.3 & \\
\hline \multicolumn{8}{|l|}{ Awareness of cervical cancer } \\
\hline Yes & 676 & 83.8 & 357 & 89.3 & 319 & 78.4 & $<0.001$ \\
\hline No & 131 & 16.2 & 43 & 10.7 & 88 & 21.6 & \\
\hline \multicolumn{8}{|l|}{ Awareness of HPV vaccination } \\
\hline Yes & 575 & 71.3 & 338 & 84.5 & 237 & 58.2 & $<0.001$ \\
\hline No & 232 & 28.7 & 62 & 15.5 & 170 & 41.8 & \\
\hline
\end{tabular}

*Household income per person was categorized into either moderate if its income $>=1,300,000 \mathrm{VND}$ or poor/near near-poor for the rest; \# Blue collar/Peasants: sales, service and craft workers, skilled labors, machine operators, and peasants; White collar: managers, professionals, experts, engineers, and office workers; and Others: students, unemployed, and housewives

urban area, about two-third self-reported as having good health regardless of their residential area. The number of women who answered that they had heard about cervical cancer was quite high $(83.8 \%)$. Although the majority of respondents were aware of cervical cancer, more than one-fifth of women living in the rural area had never heard about it prior to this survey. Additionally, women living in the urban area were more likely to be aware of cervical cancer than women in the rural area $(p<0.001)$. In contrast, nearly one-third of women answered that they had never heard about this vaccine before their participation in this study. In particular, more than $40.0 \%$ of women living in the rural area had not heard of HPV vaccination, whereas only $15 \%$ of women living in the urban area had never heard about it.

We assessed cervical cancer knowledge, after excluding 131 women who had never heard of cervical cancer prior to this study (Table 2). We found that over $40.0 \%$ of women did not know the answer or answered incorrectly for more than three out of seven questions. 
Table 2. Knowledge of Cervical Cancer by Residential Area among Women who were Aware of Cervical Cancer

\begin{tabular}{|c|c|c|c|c|c|c|c|}
\hline \multirow[t]{2}{*}{ Variables } & \multicolumn{2}{|c|}{ Overall $(n=676)$} & \multicolumn{2}{|c|}{ Urban $(n=357)$} & \multicolumn{2}{|c|}{ Rural $(\mathrm{n}=319)$} & \multirow[t]{2}{*}{$P$-value } \\
\hline & $\mathrm{n}$ & $\%$ & $\mathrm{n}$ & $\%$ & $\mathrm{n}$ & $\%$ & \\
\hline \multicolumn{8}{|l|}{ Cervical cancer is an inherited disease (False) } \\
\hline True & 171 & 25.3 & 101 & 28.3 & 70 & 21.9 & 0.16 \\
\hline False & 388 & 57.4 & 195 & 54.6 & 193 & 60.5 & \\
\hline Unknown/ No response & 117 & 17.3 & 61 & 17.1 & 56 & 17.6 & \\
\hline \multicolumn{8}{|l|}{ Cervical cancer is caused by a virus (True) } \\
\hline True & 213 & 31.5 & 147 & 41.2 & 66 & 20.7 & $<0.001$ \\
\hline False & 303 & 44.8 & 122 & 34.2 & 181 & 56.7 & \\
\hline Unknown/ No response & 160 & 23.7 & 88 & 24.6 & 72 & 22.6 & \\
\hline \multicolumn{8}{|l|}{ Cervical cancer is an infectious disease (False) } \\
\hline True & 134 & 19.8 & 72 & 20.2 & 62 & 19.4 & 0.83 \\
\hline False & 442 & 65.4 & 235 & 65.8 & 207 & 64.9 & \\
\hline Unknown/ No response & 100 & 14.8 & 50 & 14 & 50 & 15.7 & \\
\hline \multicolumn{8}{|l|}{ Population at high cervical cancer risk } \\
\hline Women ever given birth & 512 & 75.7 & 258 & 72.3 & 254 & 79.6 & 0.03 \\
\hline Older adults & 21 & 3.1 & 16 & 4.5 & 5 & 1.6 & 0.03 \\
\hline Unfaithful people & 106 & 15.7 & 54 & 15.1 & 52 & 16.3 & 0.68 \\
\hline Women with poor (gynecological) & 153 & 22.6 & 53 & 14.9 & 100 & 31.4 & $<0.001$ \\
\hline \multicolumn{8}{|l|}{ hygiene behavior } \\
\hline Point out at least one correct & 566 & 83.6 & 292 & 81.8 & 273 & 85.6 & 0.19 \\
\hline Unknown/ No response & 111 & 16.4 & 65 & 18.2 & 46 & 14.4 & \\
\hline \multicolumn{8}{|l|}{ Signs of cervical cancer } \\
\hline Abnormal vaginal bleeding & 227 & 33.6 & 75 & 21 & 152 & 47.7 & $<0.001$ \\
\hline Unusual discharge from vagina & 175 & 25.9 & 66 & 18.5 & 109 & 34.2 & $<0.001$ \\
\hline Urinary incontinence & 64 & 9.5 & 10 & 2.8 & 54 & 16.9 & $<0.001$ \\
\hline Bleeding after intercourse & 153 & 22.6 & 29 & 8.1 & 124 & 38.9 & $<0.001$ \\
\hline Pain during sexual intercourse & 118 & 17.5 & 36 & 10.1 & 82 & 25.7 & $<0.001$ \\
\hline Back pain & 52 & 7.7 & 8 & 2.2 & 44 & 13.8 & $<0.001$ \\
\hline Point out at least one sign & 331 & 49 & 122 & 34.2 & 209 & 65.5 & $<0.001$ \\
\hline Unknown/ No response & 345 & 51 & 235 & 65.8 & 110 & 34.5 & \\
\hline \multicolumn{8}{|l|}{ Cervical cancer can be prevented (True) } \\
\hline True & 618 & 91.4 & 323 & 90.5 & 295 & 92.5 & 0.15 \\
\hline False & 14 & 2.1 & 11 & 3.1 & 3 & 0.9 & \\
\hline Unknown/ No response & 44 & 6.5 & 23 & 6.4 & 21 & 6.6 & \\
\hline \multicolumn{8}{|c|}{ HPV vaccine is one of the most effective ways to prevent cervical cancer * (True) } \\
\hline True & 554 & 89.6 & 295 & 91.3 & 259 & 87.8 & 0.32 \\
\hline False & 51 & 8.3 & 23 & 7.1 & 28 & 9.5 & \\
\hline Unknown/ No response & 13 & 2.1 & 5 & 1.6 & 8 & 2.7 & \\
\hline \multicolumn{8}{|l|}{ Summary Score } \\
\hline Average score (mean, SD) & 4.6 & 1.43 & 4.51 & 1.46 & 4.71 & 1.39 & 0.07 \\
\hline Good knowledge $(\mathrm{n}, \%)(>=5 / 7$ right answers $)$ & 402 & 59.5 & 197 & 55.2 & 205 & 64.3 & 0.02 \\
\hline Not good (n,\%) (<5/7 right answers) & 274 & 40.5 & 160 & 44.8 & 114 & 35.7 & \\
\hline
\end{tabular}

*, Among women who said that cervical cancer can be prevented; SD, standard deviation

Specifically, there were many wrong answers to the following questions: "Cervical cancer is an inherited disease (right answer: false)," "Cervical cancer is caused by a virus (right answer: true)," or "Cervical cancer is an infectious disease (right answer: false)." Half did not know any signs of cervical cancer. Nevertheless, most of the participants knew that cervical cancer is preventable and that HPV vaccination is effective in preventing cervical cancer. Knowledge scores (ranging from 0-7 points) obtained by summing the number of correctly answered items in the survey are shown in Table 2. The average cervical cancer knowledge score was $4.60 \pm 1.43$ 
DOI:10.31557/APJCP.2020.21.7.1951

Knowledge of Cervical Cancer and Human Papillomavirus Vaccines among Child-Bearing Aged Women in Hanoi, Vietnam

Table 3. Knowledge of HPV Vaccination According to Residential Area among Women Aware of This Vaccine

\begin{tabular}{|c|c|c|c|c|c|c|c|}
\hline \multirow[t]{2}{*}{ Variables } & \multicolumn{2}{|c|}{ Overall $(n=575)$} & \multicolumn{2}{|c|}{$\operatorname{Urban}(\mathrm{n}=338)$} & \multicolumn{2}{|c|}{ Rural (n=237) } & \multirow[t]{2}{*}{$P$-value } \\
\hline & $\mathrm{n}$ & $\%$ & $\mathrm{n}$ & $\%$ & $\mathrm{n}$ & $\%$ & \\
\hline \multicolumn{8}{|l|}{ HPV vaccination comprises three doses in Vietnam } \\
\hline True & 96 & 16.7 & 91 & 26.9 & 5 & 2.1 & \multirow[t]{3}{*}{$<0.001$} \\
\hline False & 143 & 24.9 & 83 & 24.6 & 60 & 25.3 & \\
\hline Unknown/No response & 336 & 58.4 & 164 & 48.5 & 172 & 72.6 & \\
\hline \multicolumn{8}{|c|}{ HPV vaccines should be given at the ages of 9-26 years } \\
\hline True & 218 & 37.9 & 129 & 38.2 & 89 & 37.6 & \multirow[t]{3}{*}{$<0.001$} \\
\hline False & 159 & 27.7 & 113 & 33.4 & 46 & 19.4 & \\
\hline Unknown/No response & 198 & 34.4 & 96 & 28.4 & 102 & 43.0 & \\
\hline \multicolumn{8}{|c|}{ Women who have been sexually active can receive HPV vaccination } \\
\hline True & 263 & 45.7 & 154 & 45.6 & 109 & 46.0 & \multirow[t]{3}{*}{$<0.001$} \\
\hline False & 160 & 27.8 & 112 & 33.1 & 48 & 20.3 & \\
\hline Unknown/No response & 152 & 26.5 & 72 & 21.3 & 80 & 33.7 & \\
\hline \multicolumn{8}{|c|}{ Presence of HPV should be tested before receiving vaccination for sexually active women* } \\
\hline True & 210 & 79.9 & 126 & 81.8 & 84 & 77.1 & \multirow[t]{3}{*}{0.13} \\
\hline False & 34 & 12.9 & 21 & 13.6 & 13 & 11.9 & \\
\hline Unknown/No response & 19 & 7.2 & 7 & 4.6 & 12 & 11.0 & \\
\hline \multicolumn{8}{|l|}{ Side effect of HPV vaccines } \\
\hline Point out at least one side effect & 95 & 16.5 & 59 & 17.5 & 36 & 15.2 & \multirow[t]{3}{*}{0.001} \\
\hline No side effects & 98 & 17.0 & 73 & 21.6 & 25 & 10.6 & \\
\hline Unknown/No response & 382 & 66.5 & 206 & 60.9 & 176 & 74.2 & \\
\hline \multicolumn{8}{|l|}{ Summary Score } \\
\hline Average score (mean, SD) & 1.53 & 1.35 & 1.65 & 1.37 & 1.36 & 1.3 & 0.01 \\
\hline Good knowledge(n,\%) (>=2/5 right answers) & 286 & 49.7 & 180 & 53.3 & 106 & 44.7 & \multirow[t]{2}{*}{0.04} \\
\hline Not good (n,\%) (<2/5 right answers) & 289 & 50.3 & 158 & 46.7 & 131 & 55.3 & \\
\hline
\end{tabular}

*, Among women who said that sexually active women can receive the HPV vaccine

out of a total possible score of 7 among the 676 participants who were aware of cervical cancer. Women living in rural areas showed statistically significantly higher knowledge scores than women living in urban ones.

Table 3 shows the participants' knowledge of HPV vaccination among women who had ever heard of this vaccine. The majority of respondents showed very low levels of knowledge of HPV vaccination. More than $60.0 \%$ of respondents did know about the number of doses of HPV vaccine required in Vietnam, the ideal age for receiving the vaccine, or any side effects of HPV vaccination. Further, knowledge of HPV vaccination was significantly lower among women living in rural areas. Knowledge scores for HPV vaccination are shown in Table 3. The mean knowledge score for HPV vaccination was $1.53 \pm 1.35$ out of a total possible score of 5 . Women living in the urban area had significantly higher HPV knowledge scores than women living in the rural area.

\section{Discussion}

This is the first study to quantitatively investigate knowledge of cervical cancer and HPV vaccination among women of child-bearing age in Vietnam, where cervical cancer remains a major public health concern. This study highlights limited understanding of cervical cancer and HPV among these women who are primarily responsible for caring for their families. Therefore, tailored interventions are needed to increase public awareness and knowledge of cervical cancer and its vaccination.

As expected, the majority of respondents answered that they had heard about cervical cancer and HPV vaccination. More women said that they had heard about cervical cancer than the HPV vaccine. Similarly, previous studies have also reported greater awareness of cervical cancer than vaccination of HPV in other countries and ethnic groups (Bodson et al., 2016; Borlu et al., 2016; Saqer et al., 2017; Islam et al., 2018). This can be explained by the fact that cervical cancer is currently a great concern in many countries; however, HPV vaccines are not often covered in national immunization programs. In Vietnam, a government program implemented in 2008 was designed to cover all aspects required for the application of an HPV vaccination program; however, even though the feasibility of HPV vaccination delivery was proven, a national program for HPV vaccination in Vietnam has yet to be fully implemented. This may account for the poor awareness and knowledge of HPV vaccination among Vietnamese women.

Regarding knowledge of cervical cancer, scores for correct answers to a total of seven questions were recorded in the current study, with a mean of $4.60 \pm 1.43$ questions 
answered correctly. This mean score is much higher than reported in studies in other countries (Kietpeerakool et al., 2009; Wong and Sam, 2010; Rashwan et al., 2011; Wong, 2011; Zhao et al., 2012; Poole et al., 2013; Fernandez et al., 2014; Perlman et al., 2014; Strohl et al., 2015). Recently, with greater coverage from broadcasting programs, as well as the internet, the spread of information has become relatively easier to achieve than ever. Further, women of reproductive age might have a better knowledge of cervical cancer, as they may be more concerned with reproductive health.

Concerning HPV vaccination, the majority of respondents lacked knowledge thereof, with a mean of $1.53(\mathrm{SD}=1.35)$ questions answered correctly for a total of five questions. Similarly, low mean scores have often been reported elsewhere (Do et al., 2009; Halliday et al., 2013; Fernandez et al., 2014; Kruiroongroj et al., 2014; Perlman et al., 2014; Koc and Cinarli, 2015; Strohl et al., 2015; Bodson et al., 2016; Yu et al., 2016; Turhan et al., 2019). Furthermore, in the current study, knowledge scores for HPV vaccination were markedly lower among women living in a rural area than among those residing in an urban area. Interestingly, while the women residing in an urban area showed relatively greater HPV vaccine knowledge, they had a lower understanding of cervical cancer, compared with women who lived in a rural area. There may be several possible explanations for this: The first explanation may be related to differences in education and health promotion programs in each area. In urban areas, where various prevention services are present, education and health promotion programs for cervical cancer typically focus on HPV vaccination, whereas, in areas where prevention programs are not well organized, educational and promotional materials mainly concentrate on features and risk factors for the disease. Another explanation may be that greater cervical cancer knowledge, which was noted for women living in a rural area, might be associated with higher incidence rates of cervical cancer in rural areas (Baker et al., 2000; Benard et al., 2008).

Taking these findings together, we suggest that a comprehensive educational campaign on HPV vaccination that describes the number of required HPV vaccine shots, recommended ages for vaccination, and possible side effects is needed. Initial efforts that enable future education programs ought to be built on existing knowledge, and simultaneous attempts to address common misconceptions or unknown factors could be beneficial. Trusted persons, such as health workers or teachers, as well as influential individuals, may prove effective in disseminating such information. Meanwhile, education programs should seek to take advantage of communal health centers in order to foster community awareness, and distributing information via pamphlets or leaflets at local health centers in the countryside has been recommended as a means of increasing the acceptability of HPV vaccination in other countries (Ali, 2017).

Some limitations to this study warrant consideration. First, our data were employed in a cross-sectional study, and thus, it is beyond the present scope to determine any causal relationships. Second, the study results do not represent the entire Vietnamese population, because we targeted only two regions in this study. Lastly, this study did not include women of younger age (e.g., 12 years of age), which is the target age for receiving HPV vaccination. Additionally, the study population of pregnant women and women who recently gave birth do not represent the entire population of Vietnamese women.

Despite these limitations, our study has several strengths. First, this is the first study to investigate the associations between socio-demographic factors and levels of knowledge on cervical cancer and the HPV vaccine among Vietnamese women. Our findings indicate a need for public health policymakers to push forward step-by-step strategies for cervical cancer prevention in the near future. Second, women of child-bearing age, who are primary caretakers of children and protectors of the health for each family, were recruited, and we assessed their awareness and levels of knowledge on cervical cancer and HPV vaccine, as their knowledge about cervical cancer and HPV vaccination can strongly affect their intentions to receive vaccination themselves and for their daughters.

In conclusion, despite high levels of awareness, knowledge on cervical cancer and HPV vaccination was

lacking among women of child-bearing age in both urban and rural areas of Hanoi, Vietnam. Our findings underscore a need to develop well-designed educational programs on cervical cancer and HPV vaccination in Vietnam and other countries.

\section{Acknowledgements}

\section{Funding and Acknowledgement}

The author(s) have disclosed receipt of the following financial support for the research, authorship, and/or publication of this article. This study was part of a project, namely "Vaccination accessibility for child-bearing age women in low- and middle-income countries in Southeast Asia period 2016-2018", funded by Pfizer, an American pharmaceutical company. We thank all local guides and healthcare workers at the two District Departments of Health and Commune Health Centers for their logistic support. We thank all 20 interviewers and colleagues at Hanoi Medical University who accompanied us on the field trip and contributed to the survey.

\section{Conflicting Interests}

The author(s) have declared no conflicts of interest regarding the research, authorship, and/or publication of this article.

\section{References}

Ali AN PN, Prajapati SK, et al (2017). Development and validation of 'educational pamphlet' in prevention of human papilloma virus (HPV) infection among age eligible adults for HPV vaccination in kedah State, Malaysia. MOJ Bioequiv Availab, 4, 216-21.

Baker P, Hoel D, Mohr L, et al (2000). Racial, age, and rural/ urban disparity in cervical cancer incidence. Ann Epidemiol, 10, 466-7.

Benard VB, Johnson CJ, Thompson TD, et al (2008). Examining the association between socioeconomic status and potential 
human papillomavirus-associated cancers. Cancer, 113, 2910-8.

Bingham A, Drake JK, LaMontagne DS (2009). Sociocultural issues in the introduction of human papillomavirus vaccine in low-resource settings. Arch Pediatr Adolesc Med, 163, 455-61.

Bodson J, Warner EL, Kepka D (2016). Moderate awareness and limited knowledge relating to cervical cancer, HPV, and the HPV vaccine among Hispanics/Latinos in Utah. Health Promot Pract, 17, 548-56.

Borlu A, Gunay O, Balci E, et al (2016). Knowledge and attitudes of medical and non-medical Turkish university students about cervical cancer and HPV vaccination. Asian Pac J Cancer Prev, 17, 299-303.

Bray F, Ferlay J, Soerjomataram I, et al (2018). Global cancer statistics 2018: GLOBOCAN estimates of incidence and mortality worldwide for 36 cancers in 185 countries.

Do H, Seng P, Talbot J, et al (2009). HPV vaccine knowledge and beliefs among Cambodian American parents and community leaders. Asian Pac J Cancer Prev, 10, 339-44.

Fernandez ME, Le YC, Fernandez-Espada N, et al (2014). Knowledge, attitudes, and beliefs about human papillomavirus (HPV) vaccination among Puerto Rican mothers and daughters, 2010: a qualitative study. Prev Chronic Dis, 11, E212.

Halliday D, Butler R, Francis D, et al (2013). Knowledge and Attitudes toward HPV and the HPV Vaccines in The Bahamas. West Indian Med J, 62, 731-7.

Islam JY, Khatun F, Alam A, et al (2018). Knowledge of cervical cancer and HPV vaccine in Bangladeshi women: a population based, cross-sectional study. BMC Womens Health, 18, 15.

Kietpeerakool C, Phianmongkhol Y, Jitvatcharanun K, et al (2009). Knowledge, awareness, and attitudes of female sex workers toward HPV infection, cervical cancer, and cervical smears in Thailand. Int J Gynaecol Obstet, 107, 216-9.

Koc Z, Cinarli T (2015). Cervical cancer, human papillomavirus, and vaccination: knowledge, awareness, and practices among Turkish Hospital nurses. Nurs Res, 64, 452-65.

Kruiroongroj S, Chaikledkaew U, Thavorncharoensap M (2014). Knowledge, acceptance, and willingness to pay for human papilloma virus (HPV) vaccination among female parents in Thailand. Asian Pac J Cancer Prev, 15, 5469-74.

Perlman S, Wamai RG, Bain PA, et al (2014). Knowledge and awareness of HPV vaccine and acceptability to vaccinate in sub-Saharan Africa: a systematic review. PLoS One, 9 , e90912.

Poole DN, Tracy JK, Levitz L, et al (2013). A cross-sectional study to assess HPV knowledge and HPV vaccine acceptability in Mali. PLoS One, 8, e56402.

Prayudi PK, Permatasari AA, Winata IG, et al (2016). Impact of human papilloma virus vaccination on adolescent knowledge, perception of sexual risk and need for safer sexual behaviors in Bali, Indonesia. J Obstet Gynaecol Res, 42, 1829-38.

Rashwan H, Lubis SH, Ni KA (2011). Knowledge of cervical cancer and acceptance of HPV vaccination among secondary school students in Sarawak, Malaysia. Asian Pac J Cancer Prev, 12, 1837-41.

Saqer A, Ghazal S, Barqawi H, et al (2017). Knowledge and awareness about cervical cancer vaccine (HPV) among parents in Sharjah. Asian Pac J Cancer Prev, 18, 1237-41.

Strohl AE, Mendoza G, Ghant MS, et al (2015). Barriers to prevention: knowledge of HPV, cervical cancer, and HPV vaccinations among African American women. Am JObstet Gynecol, 212, 65.e1-5.

Tung IL, Machalek DA, Garland SM (2016). Attitudes, knowledge and factors associated with human papillomavirus (HPV) vaccine uptake in adolescent girls and young women in Victoria, Australia. PLoS One, 11, e0161846.

Turhan E, Cetin S, Cetin M, et al (2019). Awareness and knowledge levels of 18-year-old and older individuals regarding human papillomavirus (HPV) and HPV vaccine in Hatay, Turkey. J Cancer Educ, 34, 234-41.

Vaccarella S, Lortet-Tieulent J, Plummer M, et al (2013). Worldwide trends in cervical cancer incidence: impact of screening against changes in disease risk factors. Eur $J$ Cancer, 49, 3262-73.

Wong LP (2011). Knowledge and attitudes about HPV infection, $\mathrm{HPV}$ vaccination, and cervical cancer among rural southeast Asian women. Int J Behav Med, 18, 105-11.

Wong LP, Sam IC (2010). Ethnically diverse female university students' knowledge and attitudes toward human papillomavirus (HPV), HPV vaccination and cervical cancer. Eur J Obstet Gynecol Reprod Biol, 148, 90-5.

World Health Organization (2016). Human papillomavirus (HPV) and cervical cancer. [Online]. Available: http:// www.who.int/mediacentre/factsheets/fs380/en/ [Accessed Nov 10 2018].

Yu Y, Xu M, Sun J, et al (2016). Human papillomavirus infection and vaccination: Awareness and knowledge of HPV and acceptability of HPV vaccine among mothers of teenage daughters in Weihai, Shandong, China. PLoS One, 11, e0146741.

Zhao FH, Tiggelaar SM, Hu SY, et al (2012). A multi-center survey of HPV knowledge and attitudes toward HPV vaccination among women, government officials, and medical personnel in China. Asian Pac J Cancer Prev, 13, 2369-78.

\section{(0) $(\otimes)$}

This work is licensed under a Creative Commons AttributionNon Commercial 4.0 International License. 